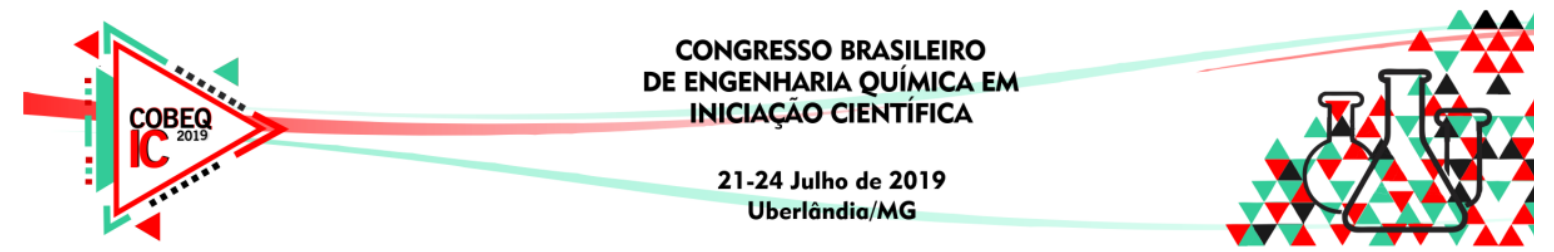

\title{
DETERMINAÇÃO DO NÚMERO DE CARBONO EFETIVO (ECN) DO FLUIDO REFRIGERANTE R-141b PARA CORREÇÃO DO FATOR DE RESPOSTA RELATIVA DO DETECTOR DE IONIZAÇÃO DE CHAMAS NA TÉCNICA DE CROMATROGRAFIA GASOSA.
}

\author{
S.L.S. NASCIMENTO ${ }^{1}$, V.M. QUEIROZ ${ }^{1}$, E.B. MARRA ${ }^{1}$, C.S.S. PEREIRA ${ }^{1}$ e S.P. \\ RIBEIRO $^{1}$ \\ ${ }^{1}$ Universidade de Vassouras, Engenharia Química \\ E-mail para contato: eq.sergionascimento@gmail.com
}

\begin{abstract}
RESUMO - O R-141b é um fluido refrigerante composto de 1,1-dicloro-1fluoroetano $\left(\mathrm{C}_{2} \mathrm{H}_{3} \mathrm{Cl}_{2} \mathrm{~F}\right)$, bastante usado para limpeza em sistema de refrigeração. Para se calcular o ECN o fluido foi analisado pela técnica analítica instrumental de separação cromatografia gasosa (CG), onde ao ser introduzido no equipamento o mesmo recebe calor para se decompor e se transformar em estado gasoso onde passa por um detector de ionização de chama, que quebra a molécula do carbono transformando em um carbocation e dando como produto dióxido de carbono $\left(\mathrm{CO}_{2}\right)$, a partícula de carbocation que dá a resposta ao detector. Como esse detector de chama é seletivo para carbono ele não detecta a cinética de combustão dos outros elementos como: Flúor (F), Cloro $(\mathrm{Cl})$, Enxofre (S),Nitrogênio $(\mathrm{N})$ etc., com isso quando ocorre a geração do carbocátion, acontece um erro experimental diferente do real. Para o acerto deste erro é preciso calcular o fator de resposta. De acordo com a norma padrão AHRI 700 para fluidos refrigerantes, o valor do número de carbonos efetivos para o R-141b não existe. Com isso, o objetivo desse trabalho foi realizar o cálculo do número efetivo de carbono para que possibilite o acerto do seu fator de resposta, e assim, avaliar se os valores são coerentes. O resultado encontrado para o número efetivo de carbono foi de aproximadamente 1,60 e com isso foi possível calcular o fator de resposta demonstrando satisfatoriedade da metodologia aplicada.
\end{abstract}

\section{INTRODUÇÃO}

As técnicas analiticas de separção, tais como a CG se baseiam na separação de espécies químicas voláteis que percolam por dentro de uma coluna específica para cada amostra e através da separação dessas espécies, ao final da mesma são gerados cromatogramas onde são analisados as áreas entre os picos onde se compara o valor teórico com o experimental.

De acordo com a (Dupont fluorproducts) o R-141b se tornou uma alternativa provisória aceitável para o fluido refrigerante CFC-11. Em aplicações de espuma rígida, tais como: construção, eletrodomésticos e veículos de transporte (GMALATO 2007/2016). O CFC-11 foi muito utilizado na decada de 80 e 90 principalmente na indústria de produtos de refrigeração, 


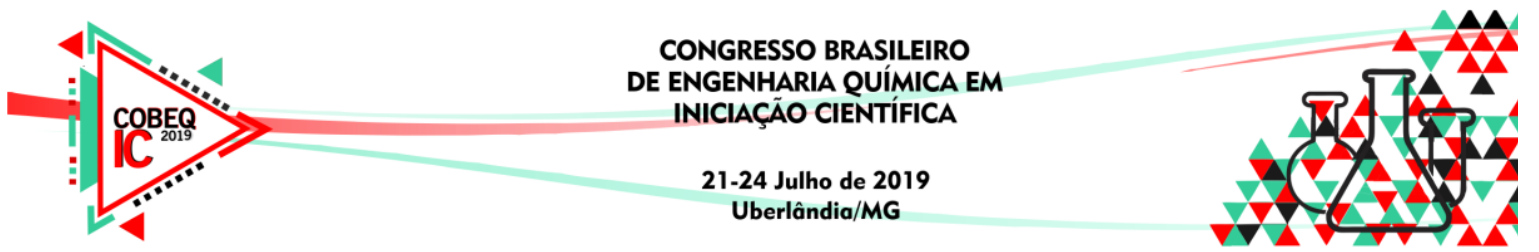

mas por ser a principal substância destruidora da camada de Ozônio, o consumo foi banido em 2010.

O trabalho de Scanlon, e Willis (1985), cita que é desejavél ter alguns meios para calcular o fator de resposta para se comparar com o valor experimental, para isso se utiliza o detector de ionização de chama (FID). O conceito de número efetivo de carbono (ECN) é utilizado para analisar uma série homologa de compostos orgânicos na separação da reação ao formar o carbocátion, e foi introduzido a anos atrás para se explicar a resposta de ionização de chamas desses compostos.

Com a verificação da norma padrão utilizada para fluidos refrigerantes ARHI 700, não é encontrado o valor para o ECN do R-141b. Por esse motivo, o presente trabalho visa calcular o fator de resposta para o cálculo do ECN e possibilitar os cálculos para o fator de correção do fluido.

\section{MATERIAIS E MÉTODOS}

Todos os dados foram obtidos usando um cromatrografo a gás modelo HP 6890 Plus, a

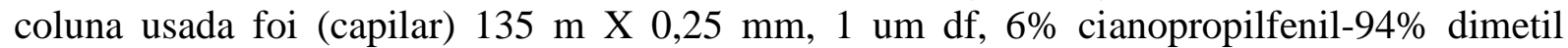
polissiloxano. Como gás de arraste foi utilizado o hélio $(\mathrm{He})$. Para a ionização da chama foi utilizado os gases ar sintético e hidrogênio. Para o sistema de criogenia foi utilizado o fluido $\mathrm{CO}_{2}$.

Conforme o AHRI 700, pode-se visualizar o método utilizado para a análise cromatográfica (tabela 1).

Tabela 1 - Método utilizado para a análise cromatográfica do fluido R-141b

\begin{tabular}{|c|c|}
\hline \multicolumn{2}{|c|}{ Condiç̃os de operação CG } \\
\hline Condição & R-141b \\
\hline Detector & FID \\
\hline Gás portator, cc Hélio por minuto & 1.0 \\
\hline Temperatura da porta de injeção, ${ }^{\circ} \mathrm{C}\left[{ }^{\circ} \mathrm{F}\right]$ & $200[392]$ \\
\hline Temperatura do detector, ${ }^{\circ} \mathrm{C}\left[{ }^{\circ} \mathrm{F}\right]$ & $200[392]$ \\
\hline Temperatura inicial da coluna, ${ }^{\circ} \mathrm{C}\left[{ }^{\circ} \mathrm{F}\right]$ & $10[50]$ \\
\hline Espera inicial, min & 12 \\
\hline Programa 1 & $10[50]$ \\
Rampa $={ }^{\circ} \mathrm{C} / \min \left[{ }^{\circ} \mathrm{F} / \mathrm{min}\right]$ & $100[212]$ \\
Temperatura da coluna, ${ }^{\circ} \mathrm{C}\left[{ }^{\circ} \mathrm{F}\right]$ & 5.00 \\
Manter, minutos & $15[59]$ \\
Programa 2 & $150[302]$ \\
Rampa $={ }^{\circ} \mathrm{C} / \mathrm{min}\left[{ }^{\circ} \mathrm{F} / \mathrm{min}\right]$ & 6.67 \\
\hline Temperatura da coluna, ${ }^{\circ} \mathrm{C}\left[{ }^{\circ} \mathrm{F}\right]$ & 36 \\
\hline Manter, minutos & 40 por 1 \\
\hline Tempo total de execução, min & $\mathrm{CO}$ \\
\hline Relação de divisão & $280[536]$ \\
\hline Resfriamento subambiente & \\
\hline Temperatura máxima segura da coluna, ${ }^{\circ} \mathrm{C}\left[{ }^{\circ} \mathrm{F}\right]$ & \\
\hline
\end{tabular}




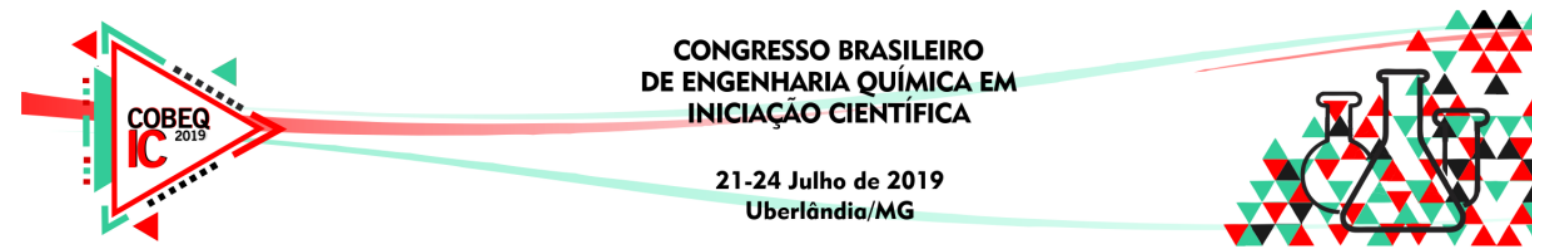

O primeiro passo para realizar a análise foi preparar um padrão de R-141b juntamente com um hidrocarboneto de referência, para isso utilizou-se dois produtos, conforme mostrado abaixo:

1) Uma ampola padrão de $1 \mathrm{~mL}$ de $141 \mathrm{~b}$ com $1004,7 \mathrm{ug} / \mathrm{mL}$ diluído em metanol, de acordo com o certificado do material (Absolute Standards)

2) $2 \mathrm{uL}$ de n-hexano com $95 \%$ de pureza.

Os produtos 1 e 2, foram misturados tendo assim no padrão o 141b 1004,7 ug/mL, a quantidade (em ug) de n-hexano adicionada foi encontrada pelo cálculo abaixo:

A densidade do n-hexano a $25^{\circ} \mathrm{C}$ é $659 \mathrm{ug} / \mathrm{uL}$, então foi adicionado $1318 \mathrm{ug}$ de n-hexano. Como a pureza do n-hexano era $95 \%$, multiplicou-se $1318 \times 0,95=1252,1$ ug de $n$-hexano.

Dessa forma foi possível encontrar as quantidades de n-hexano e $141 \mathrm{~b}$ no padrão, em seguida a mistura foi injetada em duplicata no cromatógrafo para testes, não sendo necessário mais medidas pois com o valor da média encontrada nessas duas análises já foi suficiente para corrigir o fator resposta através do cálculo do ECN.

O uso apenas do metanol diluido no R141b foi devido aos procedimentos rotineiros, comuns, nessas análises, com a aplicabilidade apenas desse hidrocarboneto.

\section{RESULTADOS E DISCUSSÕES}

Ao injetar a amostra, dentro do cromatógrafo ocorre uma separação dos compostos orgânicos e o calor da chama que é composta de $\mathrm{H}_{2}$ e $\mathrm{O}_{2}$ quebra a cadeia reagindo com o carbono para formar carbocátion e posteriormente dióxido de carbono $\left(\mathrm{CO}_{2}\right)$. Como FID é seletivo para carbono, as vazões dos gases e a geometria do detector não consegue detectar a cinética de combustão dos outros elementos como: Flúor (F), Cloro $(\mathrm{Cl})$, Enxofre $(\mathrm{S})$, Nitrogênio $\left(\mathrm{N}_{2}\right)$, entre outros. A partícula de carbocátion que remete a resposta ao FID. Em razão disso ao final da separação na formação dos compostos a porcentagem da área não coincide com o teor real, e para corrigir esse erro é preciso calcular os fatores de resposta dos elementos.

Um cromatograma conforme a Figura 1, foi gerado ao final da separação dos fluidos e os valores das aréas foram encontrados e são de suma importância para os cálculos e correção do mesmo. 


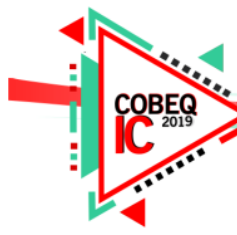

CONGRESSO BRASILEIRO

DE ENGENHARIA QUIMICA EM

INICIAÇÃO CIENTÍFICA

21-24 Julho de 2019

Uberlândia/MG

Figura 1 - Cromatograma da primeira análise.

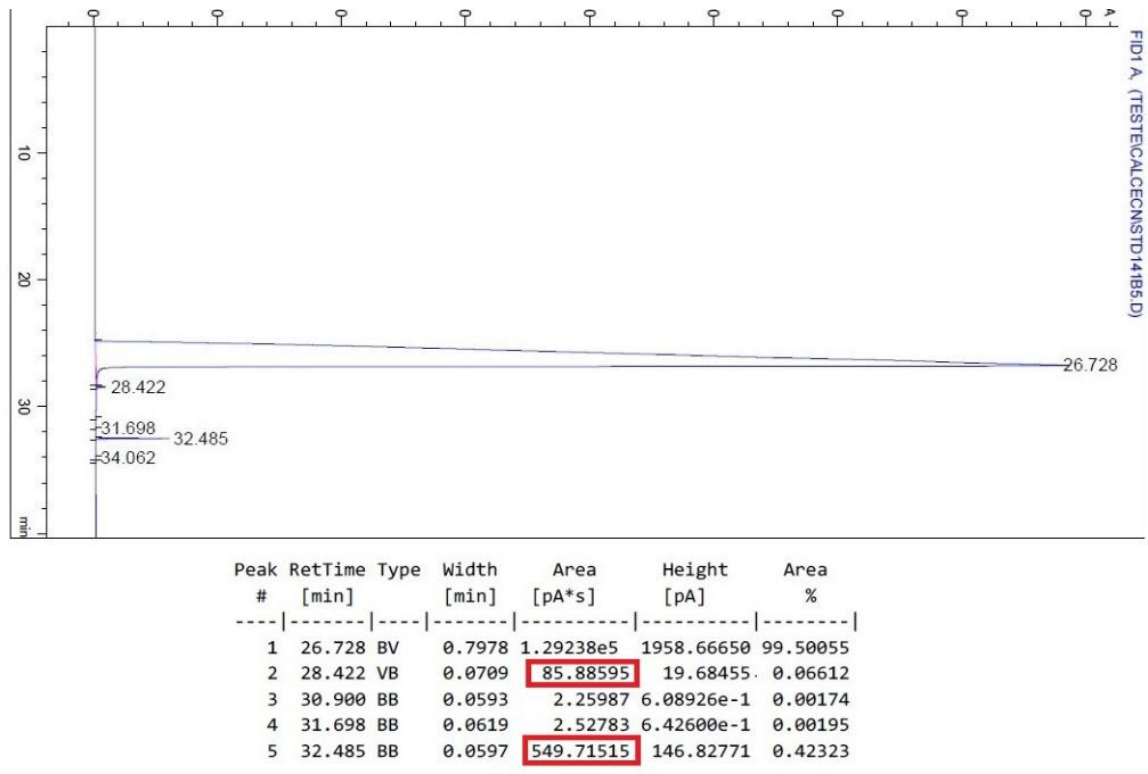

$\mathrm{Na}$ segunda análise, no resultado do cromatograma os picos dos fluidos foram encontrados com uma pequena diferença em relação a primeira análise, e foram encontrados mais picos possivelmente de interferências.

Figura 2 - Cromatograma da segunda análise.

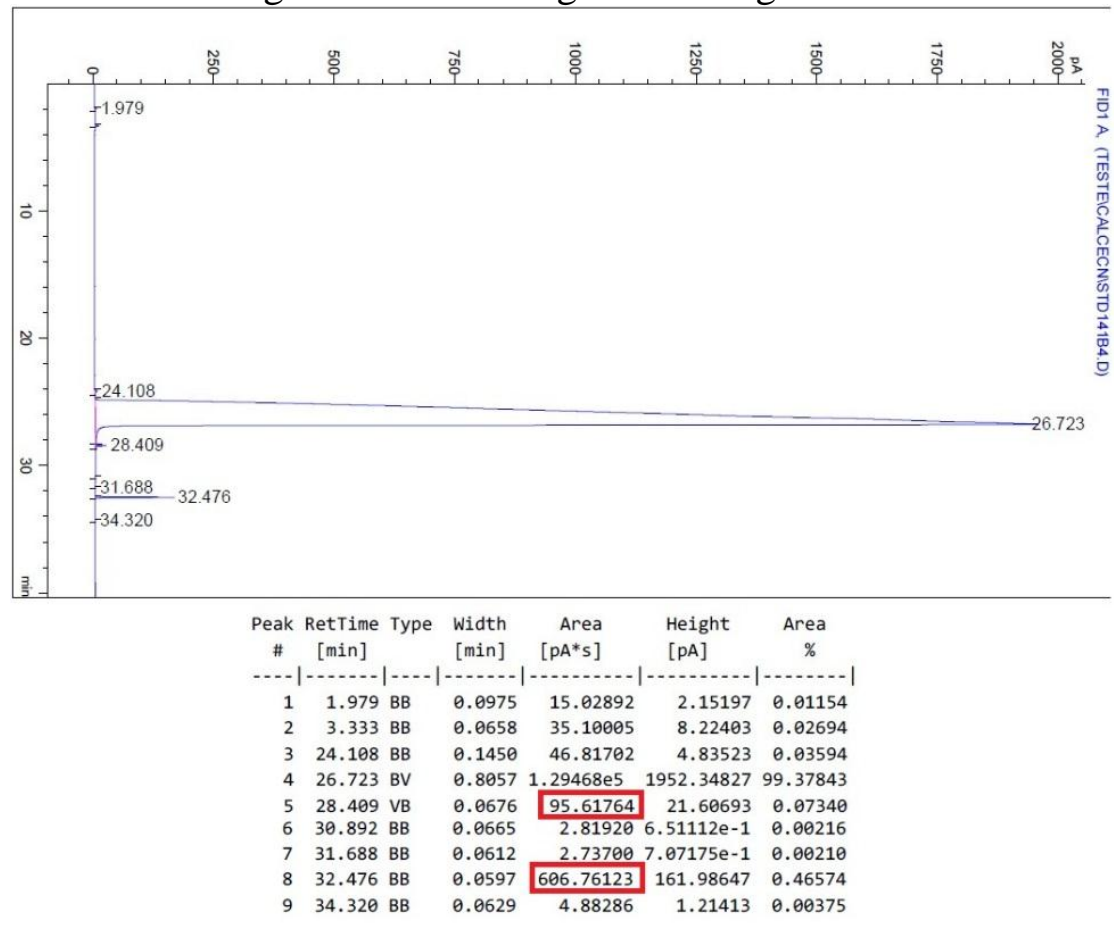

Conforme mostrado nas Figuras 1 e 2, as áreas e as massas encontradas em duplicata, bem como a média respectivamente e o cálculo de ECN para o componente R-141b empregando a Equação 2 podem ser visualizados conforme a Tabela 2. 


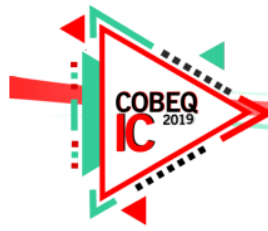

Tabela 2 - Resultados dos cálculos realizados para a obtenção de um valor de ECN para o fluído refrigerante R-141b

\begin{tabular}{|c|c|c|c|c|c|}
\hline Componente & Área 1 $(\mathbf{p A}$ *s) & Área 2 $(\mathbf{p A} * \mathbf{s})$ & Média(Áreas) & $\begin{array}{c}\text { Média das } \\
\text { massas }\end{array}$ & ECN \\
\hline \hline n-hexano & 549,7 & 606,80 & 578,25 & 32,48 & 6 \\
\hline R-141b & 85,9 & 95,60 & 90,75 & 26,72 & 1,557 \\
\hline
\end{tabular}

Usando média das áreas e massas, calculamos o fator de resposta, em peso do R-141b:

$F R=\frac{\text { área do } \mathrm{n}-\text { hexano } \mathrm{x} \text { massa do } \mathrm{R}-141 \mathrm{~b}}{\text { área do } R-141 \text { b } \times \text { massa do } n-\text { hexano }}$

$F R=5,242$

Com esse fator (FR), calculou-se o ECN do R-141b.

ECC do componente $=\frac{\text { Peso Molar do } 141 b * \text { ECN do } n-\text { Hexano }}{\text { Peso Molar do } n-H e x a n o * F R(R-141 b)}$

Peso Molar do 141b: $117 \mathrm{~g} / \mathrm{mol}$

Peso Molar do n-Hexano: $86 \mathrm{~g} / \mathrm{mol}$

ECN: 1,557, aproximadamente 1,60.

Então usando o valor de ECN calculado do fluido R-141b foi possível calcular o fator de resposta em porcentagem do peso do mesmo e através da formula abaixo como o valor do ECN dos demais compostos foi encontrado na norma AHRI 700 (2014) foram calculados os fatores de resposta para todos os fluidos conforme Tabela 3:

$F R=\frac{\text { Peso Molar do }(R-141 b) * E C N \text { do } n-\text { Hexano }}{\text { Peso Molar do n-Hexano } * \text { ECN do }(R-141 b)}$

$F R(R-141 b)=5,101744$

Tabela 3 - Fatores de resposta dos fluidos.

\begin{tabular}{|c|c|c|c|}
\hline Produtos & ECN & Peso Molar $(\mathbf{g} / \mathbf{m o l})$ & FR \\
\hline R-23 & $0,16^{*}$ & 70 & 30,523260 \\
\hline R-32 & $0,62^{*}$ & 52 & 5,851463 \\
\hline R-1123 & $1,93^{*}$ & 82 & 2,964213 \\
\hline R-143 & $2,12^{*}$ & 84 & 2,764370 \\
\hline R-125 & $0,79^{*}$ & 120 & 10,597590 \\
\hline R-115 & $0,76^{*}$ & 154 & 14,137090 \\
\hline R-1243zf & $2,84^{*}$ & 96 & 2,358336 \\
\hline R-12 & $0,35^{*}$ & 121 & 24,119600 \\
\hline R-1122 & $1,76^{*}$ & 98 & 3,884778 \\
\hline R-124 & $1,33^{*}$ & 136 & 7,134114 \\
\hline R-31 & $0,92^{*}$ & 68 & 5,156724 \\
\hline
\end{tabular}




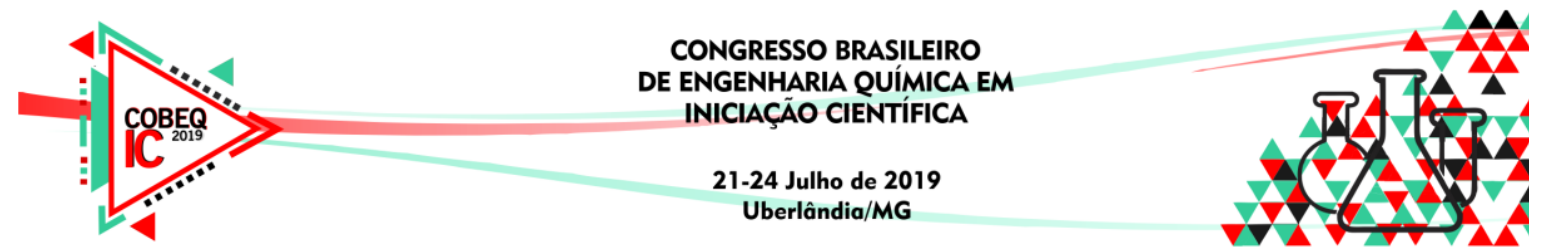

\begin{tabular}{|c|c|c|c|}
\hline R-133a & $1,93^{*}$ & 118 & 4,265574 \\
\hline R-1336mzz & $2,90^{*}$ & 164 & 3,945469 \\
\hline R-114 & $1,04^{*}$ & 170 & 11,404290 \\
\hline R-114a & $1,10^{*}$ & 170 & 10,782240 \\
\hline R-11 & $0,43^{*}$ & 137 & 22,228230 \\
\hline R-1112a & $1,64^{*}$ & 133 & 5,657969 \\
\hline R-1121C & $1,75^{*}$ & 115 & 4,584718 \\
\hline R-123 & $1,76^{*}$ & 153 & 6,065011 \\
\hline R-1121T & $1,75^{*}$ & 115 & 4,584718 \\
\hline R-113 & $1,60^{*}$ & 188 & 8,197674 \\
\hline R-134 & $1,61^{*}$ & 102 & 4,420049 \\
\hline R-152a & $1,08^{*}$ & 66 & 4,263566 \\
\hline R-1234yf & $2,65^{*}$ & 114 & 3,001316 \\
\hline R-141b & 1,60 & 117 & 5,101744 \\
\hline
\end{tabular}

* Valores de ECN encontrados na norma AHRI 700 (2014).

\section{CONCLUSÃO}

Ao final das análises, foi possível destacar que é de suma importância para o desenvolvimento da técnica instrumental de separação cromatografia gasosa calcular os fatores de resposta para análises utilizando o detector de ionização de chama (FID), corrigindo o erro das moléculas que possuem ( $\mathrm{F}, \mathrm{Cl}, \mathrm{S}, \mathrm{N}$.) entre outros, que não irão formar carbocation, e não irão apresentar a porcentagem área com o teor real. Sem os cálculos de ECN e FR a análise de composição das impurezas e do seu produto principal é imprecisa devido o detector FID necessitar da resposta do carbocátion.

\section{REFERENNCIAS}

ABSOLUTE STANDARDS, INC. Certified Reference Material CRM - Certified Weight Report, 2017.

AHRI STANDARD 700-2014.; 2008 Appendix C for Analytical Procedures for AHRI Standard 700-2014-Normative, 2014.

DUPONT FLUORPRODUCTS.; (Dupont HCFC-141b properties, uses, storage and handling).

GMALATO. Fluidos refrigerantes - Aplicações, 2007-2016. Disponível em: <http://www.ambientegelado.com.br/v51/index.php/artigostecnicos/fluidosrefrigerantes /tipos-de-fluidos/368-fluidos-refrigerantes-aplicacoes>. Acesso em: 10 mar. 2019.

SCANLON, J. T.; WILLIS, D. E.; Calculation of flame Ionization Detector Relative Response Factors Using the Effective Carbon Number Concept.; Journal of Chromatographic Science.; v. 23, August, 1985. 